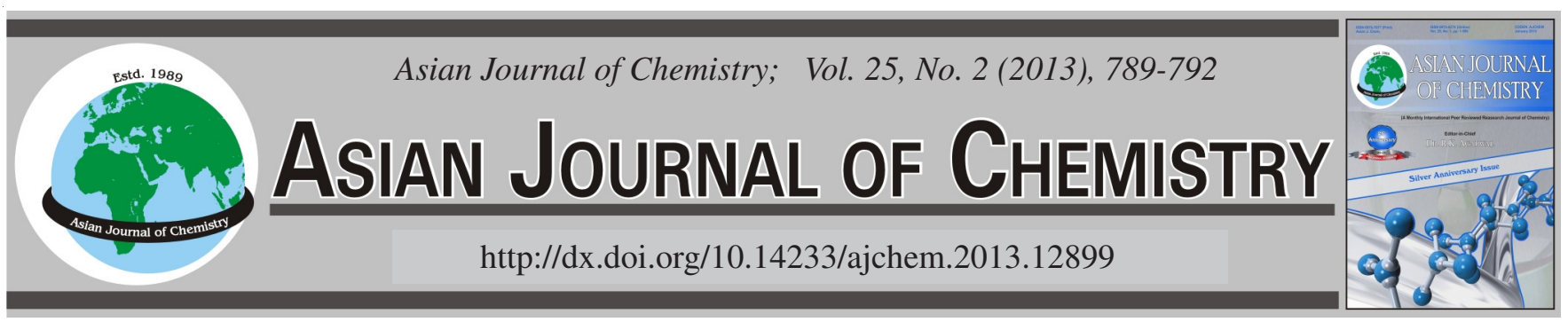

\title{
Synthesis, Characterization and Biological Evaluation of Some New Isoxazoline Derivatives
}

Dinesh K. Jain ${ }^{*}$, NeERAJ Goyal and Upendra Bhadoriya

College of Pharmacy, IPS Academy, Indore-452 012, India

*Corresponding author: E-mail: dk57@ rediffmail.com

\begin{abstract}
Isoxazoline represents a unique class of nitrogen- and oxygen-containing five-membered heterocycles, a class of compounds of great importance in biological chemistry. Isoxazoline is considered as one of the most potent antimicrobial compound. Isoxazoline derivatives have been found to possess appreciable antitubercular activity against Mycobacterium tuberculosis. The object of antitubercular drug discovery has become a subject of greater challenge due to increasing incidences of tubercular drug resistance. The study aimed to synthesize potent antitubercular isoxazline derivatives. Different substituted isoxazoline derivatives have been synthesized by cyclization of substituted chalcone derivatives in presence of hydroxylamine hydrochloride. Synthesized derivatives were characterized by melting point, TLC, FT-IR, ${ }^{1} \mathrm{H}$ NMR and MS spectroscopy. Synthesized conjugates were evaluated for in vitro antitubercular activity against M. tuberculosis. Comparable antitubercular activity was obtained as compared to isoniazide.
\end{abstract}

Key Words: Isoxazoline, Antitubercular activity, Chalcone, Hydroxylamine hydrochloride.

\section{INTRODUCTION}

Tuberculosis is caused by Mycobacterium tuberculosis, a deadly obligate bacterial pathogen. The major concerns for current tuberculosis treatment are its latency, co-infection with $\mathrm{HIV}$, poor patient compliance and drug resistance issues caused by the emergence of multidrug resistant tuberculosis (MDRTB) and the recent advent of extensively drug resistant tuberculosis (XDR-TB). Streptomycin was introduced in 1944 for the treatment of tuberculosis which was followed by first line tuberculosis drugs such as isoniazid, rifampicin, ethambutol and pyrizinamide and later by second line of drugs ${ }^{1}$. Multidrug regimen was found to be effective for the treatment of tuberculosis (isoniazid + rifampicin + pyrizinamide + ethambutol). MDR is observed in all front line and second line of drugs. Resistance toward isoniazid has arisen due to mutation of katG and inhA, resistance toward rifampicin due to mutation of rpoB-gene, resistance toward pyrizinamide due to mutation of pncA gene, etc. Hence, there is a need to develop potent and fast acting antituberculosis drugs with new modes of action to overcome the cross-resistance with current drugs and low toxicity profiles that can be tolerated for long treatment periods required for tuberculosis chemotherapy ${ }^{2}$. In an effort to develop novel antituberculosis therapeutics, we reported two series of isoxazoline compounds through modification of the 3,5-disubstituted isoxazoline core with potent inhibitory activity against $M$. tuberculosis. The isoxazoline derivatives were synthized via cyclization of substituted chalcone intermediate in presence of hydroxylamine hydrochloride ${ }^{3,4}$.

The purpose of this study was to develop new isoxazoline derivatives as potent antituberculer agents against $M$. tuberculosis.

\section{EXPERIMENTAL}

Melting points were determined by thieles tube (Table-1) and were uncorrected. ${ }^{1} \mathrm{H}$ NMR spectra were recorded on Bruker Avance II 400 NMR spectrometer. FT-IR spectra were recorded on Perkin Elmer 1600 and Mass spectra were recorded on Jeol SX-102 mass spectrometer.

\section{Synthesis of substituted isoxazoline derivatives}

Procedure for synthesis of synthesis of substituted chalcone derivative:

Claisen-Schmidt condensation: A solution of sodium hydroxide (40\%) in water and rectified spirit was placed in a flask provided with a mechanical stirrer. The flask was immersed in a bath of crushed ice. Substituted acetophenone $(0.006 \mathrm{M})$ was poured with stirring, then substituted benzaldehyde $(0.006 \mathrm{M})$ was added. The temperature of the mixture was kept at ca. $25^{\circ} \mathrm{C}$ (the limits being $15-30{ }^{\circ} \mathrm{C}$ ) and was stirred vigorously until the mixture was so thick that stirring was no longer effective (4-6 h). The stirrer was removed and the reaction mixture was left in refrigerator overnight. The product was filtered with suction on a buchner funnel, washed 
TABLE-1

COMPOUNDS CODE, STRUCTURE AND IUPAC NAMES OF THE SYNTHESIZED SUBSTITUTED ISOXAZOLINE DERIVATIVES

Conjugate code

$\mathrm{NA}_{1}$<smiles>O=[N+]([O-])c1cccc(C2=NOC(c3ccc(Br)cc3)C2)c1</smiles>

$\mathrm{NA}_{2}$<smiles>O=[N+]([O-])c1cccc(C2=NOC(c3ccc(Cl)cc3)C2)c1</smiles>

$\mathrm{NA}_{3}$

$\mathrm{NA}_{4}$

$\mathrm{NA}_{5}$<smiles>O=[N+]([O-])c1cccc(C2=NOC(c3ccc(O)cc3)C2)c1</smiles>

$\mathrm{O}_{2}$<smiles>CN(C)c1ccc(C2CC(c3cccc([N+](=O)[O-])c3)=NO2)cc1</smiles>

$\mathrm{NA}_{6}$

$\mathrm{NB}_{1}$<smiles>COc1ccc(C2CC(c3cccc([N+](=O)[O-])c3)=NO2)cc1</smiles><smiles>Cc1ccc(C2CC(c3cccc(N)c3)=NO2)cc1</smiles>

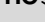<smiles>Oc1cccc(C2=NOC(c3ccc(Br)cc3)C2)c1</smiles>

$\mathrm{NB}_{2}$<smiles>Oc1cccc(C2=NOC(c3ccc(Cl)cc3)C2)c1</smiles>

$\mathrm{NB}_{3}$
IUPAC name of conjugates

5-(4-Bromophenyl)-3-(3-nitrophenyl)-4,5dihydroisoxazole

5-(4-Chlorophenyl)-3-(3-nitrophenyl)-4,5dihydroisoxazole

4-(3-(3-Nitrophenyl)-4,5-dihydroisoxazol-5yl)phenol

N,N-Dimethyl-4-(3-(3-nitrophenyl)-4,5dihydroisoxazol-5-yl)benzenamine

5-(4-Methoxyphenyl)-3-(3-nitrophenyl)-4,5dihydroisoxazole

3-(3-Nitrophenyl)-5-p-tolyl-4,5dihydroisoxazole

3-(5-(4-Bromophenyl)-4,5-dihydroisoxazol-3yl)phenol

3-(5-(4-Chlorophenyl)-4,5-dihydroisoxazol-3yl)phenol

3-(5-(4-Hydroxyphenyl)-4,5-dihydroisoxazol3 -yl)phenol 


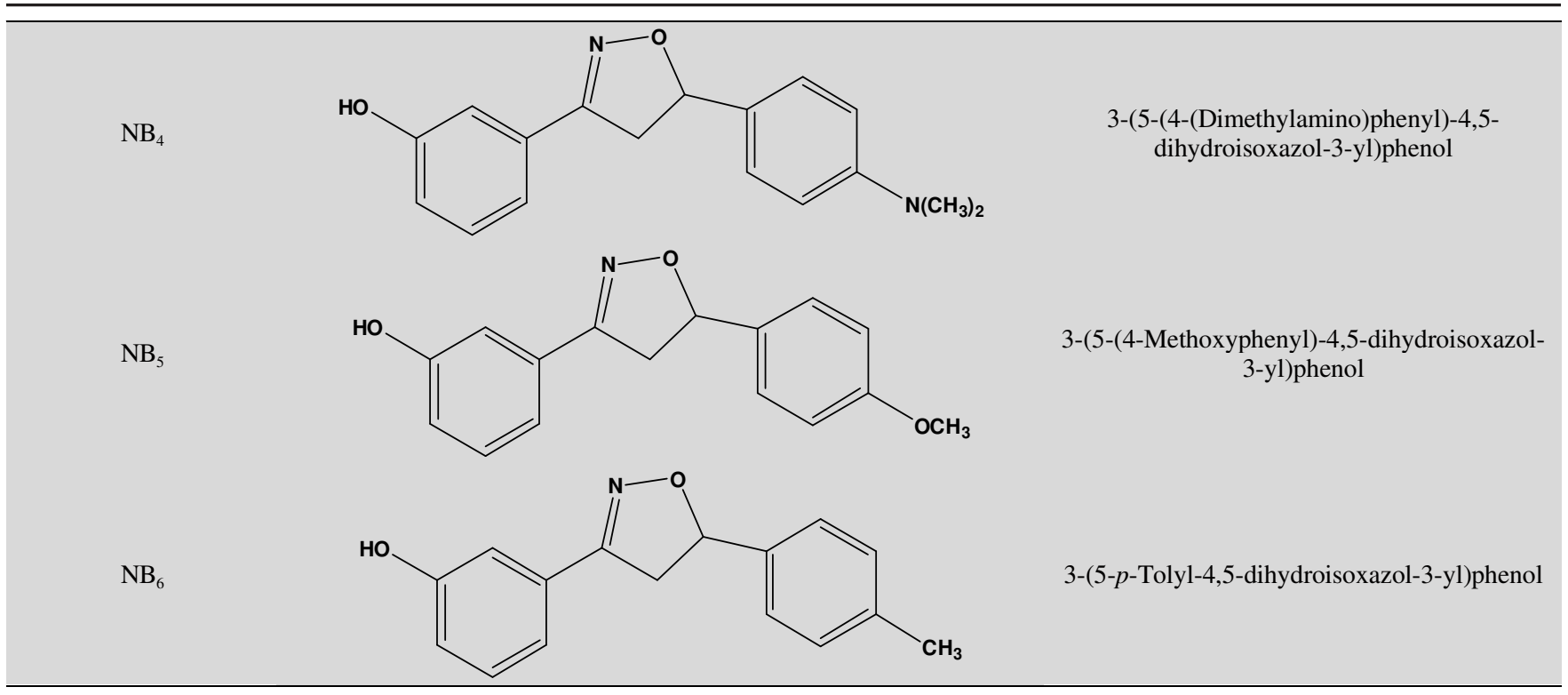

with cold water until the washings were neutral to litmus and then with ice cold ethanol. The crude product was recrystallized from ethanol (i).

Addition and cyclization: A mixture of chalcone and hydroxylamine hydrochloride in ethanol was taken in a round bottom flask. The reaction mixture was refluxed for $6 \mathrm{~h}$ on a water bath and then ice cold water was added to it at room temperature. The mixture was kept overnight in the freezer. The precipitates were filtered, washed with distilled water and dried. The product was recrystallized with ethanol to afford pure crystals (ii) (Scheme-I).<smiles>[2H]c1cccc(C(C)=O)c1</smiles>

$m$-Substituted acetophenone<smiles>O=Cc1ccc(Br)cc1</smiles>

Substituted benzaldehyde<smiles>[R]c1ccc(/C=C/C(=O)c2cccc(P)c2)cc1</smiles>

Chalcone (i)<smiles>[R]c1ccc(C2CC(c3cccc([R])c3)=NO2)cc1</smiles>

$\left(\mathrm{R}=\mathrm{NO}_{2}\right.$ or $\left.\mathrm{OH}\right)$

Scheme-I: Synthesis of 3-(3-substituted phenyl)-5-phenyl-4,5-dihydroisoxazole substituted isoxazoline

\section{Biological evaluation}

In vitro antitubercular activity of synthesized derivatives: All chemicals were dissolved in DMSO (100 mg/1 mL) and different dilutions of chemicals in each well were filled, such as 2.5, 5.0, 7.5 and $10 \mathrm{mg}$. Control- DMSO as a control. (--) indicate as negative (none zone).

Kirby-Bauer disk diffusion method for antibacterial stability test: The standard Kirby-Bauer disk diffusion method was used to determine the antimicrobial chemicals. The $M$. tuberculosis strain were inoculated in nutrient broth and incubated at $37^{\circ} \mathrm{C}$ for overnight. After overnight incubation, the bacterial culture was swapped in solidified Muller hinton agar plate and made wells of $6 \mathrm{~mm}$ diameter were punched in the plate. Then add different concentration of chemicals was dispensed in separate wells such as 2.5, 5.0, 7.5 and $10 \mathrm{mg}$ and add $100 \mu \mathrm{L}$ of DMSO to centre well as control. The plates were incubated at $37^{\circ} \mathrm{C}$ for $24 \mathrm{~h}$. After incubation, the diameter of zone of inhibition around the wells were measured and recorded.

Composition of Muller Hinton Agar: Ingredients g/L. Beef extract $-2.0 \mathrm{~g}$, starch - $1.5 \mathrm{~g}$, acid hydrolysate of casein - $17.5 \mathrm{~g}$, Agar - $17.0 \mathrm{~g}$, final $\mathrm{pH}-7.3 \pm 0.1$ at $25^{\circ} \mathrm{C}$.

Composition of nutrient broth: Ingredients $\mathrm{g} / \mathrm{L}$, peptic digest of animal tissue $-5 \mathrm{~g}$, sodium chloride $-5 \mathrm{~g}$, Beef extract - $1.5 \mathrm{~g}$, yeast extract $-1.5 \mathrm{~g}$, final $\mathrm{pH}\left(\right.$ at $\left.25^{\circ} \mathrm{C}\right)-7.4 \pm 0.2$.

\section{RESULTS AND DISCUSSION}

Substituted isoxazoline derivatives were synthesized using substituted acetophenones and substituted benzaldehydes via cyclization of substituted chalcone. 12 such compounds were synthesized. They were evaluated for their in vitro antitubercular activity against $M$. tuberculosis. They showed improved antitubercular activity over the parent drug. The results are mentioned in Table-2.

Structure of all the synthesized conjugates have been established on the basis of their consistent IR, ${ }^{1} \mathrm{H}$ NMR and mass spectral data. The synthesized conjugates showed the presence of amide and ester functional group along with the presence of aromatic and aliphatic ring which was also evident in the ${ }^{1} \mathrm{H}$ NMR spectra of the synthesized conjugates (Table-3). 


\begin{tabular}{ccccc}
\hline \multicolumn{5}{c}{ TABLE-2 } \\
\multicolumn{5}{c}{$\begin{array}{c}\text { PHYSICAL CONSTANTS OF SYNTHESIZED } \\
\text { ISOXAZOLINE DERIVATIVES }\end{array}$} \\
\hline $\begin{array}{c}\text { Conjugate } \\
\text { code }\end{array}$ & m.f. & m.w. & m.p. $\left({ }^{\circ} \mathrm{C}\right)$ & $\mathrm{R}_{\mathrm{f}}^{*}$ value \\
\hline $\mathrm{NA}_{1}$ & $\mathrm{C}_{15} \mathrm{H}_{11} \mathrm{~N}_{2} \mathrm{O}_{3} \mathrm{Br}$ & 347 & $110-115$ & 0.67 \\
$\mathrm{NA}_{2}$ & $\mathrm{C}_{15} \mathrm{H}_{11} \mathrm{~N}_{2} \mathrm{O}_{3} \mathrm{Cl}$ & 302 & $135-140$ & 0.73 \\
$\mathrm{NA}_{3}$ & $\mathrm{C}_{15} \mathrm{H}_{12} \mathrm{~N}_{2} \mathrm{O}_{4}$ & 284 & $90-95$ & 0.69 \\
$\mathrm{NA}_{4}$ & $\mathrm{C}_{17} \mathrm{H}_{17} \mathrm{~N}_{3} \mathrm{O}_{3}$ & 311 & $100-105$ & 0.81 \\
$\mathrm{NA}_{5}$ & $\mathrm{C}_{16} \mathrm{H}_{14} \mathrm{~N}_{2} \mathrm{O}_{4}$ & 298 & $120-125$ & 0.83 \\
$\mathrm{NA}_{6}$ & $\mathrm{C}_{16} \mathrm{H}_{14} \mathrm{~N}_{2} \mathrm{O}_{3}$ & 282 & $80-85$ & 0.79 \\
$\mathrm{NB}_{1}$ & $\mathrm{C}_{15} \mathrm{H}_{12} \mathrm{NO}_{2} \mathrm{Br}$ & 318 & $120-125$ & 0.84 \\
$\mathrm{NB}_{2}$ & $\mathrm{C}_{15} \mathrm{H}_{12} \mathrm{NO}_{2} \mathrm{Cl}$ & 273 & $135-140$ & 0.83 \\
$\mathrm{NB}_{3}$ & $\mathrm{C}_{15} \mathrm{H}_{13} \mathrm{NO}_{3}$ & 255 & $200-205$ & 0.72 \\
$\mathrm{NB}_{4}$ & $\mathrm{C}_{17} \mathrm{H}_{18} \mathrm{~N}_{2} \mathrm{O}_{2}$ & 282 & $120-125$ & 0.74 \\
$\mathrm{NB}_{5}$ & $\mathrm{C}_{16} \mathrm{H}_{15} \mathrm{NO}_{3}$ & 269 & $115-120$ & 0.66 \\
$\mathrm{NB}_{6}$ & $\mathrm{C}_{16} \mathrm{H}_{15} \mathrm{NO}_{2}$ & 253 & $155-160$ & 0.56 \\
\hline
\end{tabular}

\begin{tabular}{|c|c|c|}
\hline \multicolumn{3}{|c|}{$\begin{array}{c}\text { TABLE-3 } \\
\text { SPECTRAL DATA OF THE SYNTHESIZED DERIVATIVES }\end{array}$} \\
\hline $\begin{array}{l}\text { Comp. } \\
\text { code }\end{array}$ & $\begin{array}{l}\text { Mass spectra } \\
\text { (molecular } \\
\text { ion peak) }\end{array}$ & ${ }^{1} \mathrm{H}$ NMR spectra $(\delta)$ in ppm \\
\hline $\mathrm{NA}_{1}$ & 347.99 & $\begin{array}{l}\text { 7.61-8.51 (r, } 4 \mathrm{H}, \text { aromatic ring }), 7.08-7.36 \\
(\mathrm{~s}, 4 \mathrm{H}, \text { aromatic ring), } 4.5(\mathrm{~m}, 1 \mathrm{H}, \mathrm{CH}) \text {, } \\
3.39-3.52\left(\mathrm{~s}, 2 \mathrm{H}, \mathrm{CH}_{2} \text { in ring) }\right.\end{array}$ \\
\hline $\mathrm{NA}_{2}$ & 303.05 & $\begin{array}{l}\text { 7.61-8.51 (r, } 4 \mathrm{H} \text {, aromatic ring), } 7.13-7.20 \\
(\mathrm{~s}, 4 \mathrm{H}, \text { aromatic ring), } 4.5(\mathrm{~m}, 1 \mathrm{H}, \mathrm{CH}) \text {, } \\
3.39-3.52\left(\mathrm{~s}, 2 \mathrm{H}, \mathrm{CH}_{2} \text { in ring }\right)\end{array}$ \\
\hline $\mathrm{NA}_{3}$ & 285.08 & $\begin{array}{l}7.61-8.49(\mathrm{r}, 4 \mathrm{H}, \text { aromatic ring), } 7.77-8.02 \\
(\mathrm{s}, 4 \mathrm{H} \text {, aromatic ring), } 7.57(\mathrm{r}, \mathrm{OH}), 3.37(\mathrm{~s}, \\
2 \mathrm{H}, \mathrm{CH}_{2} \text { in ring) }\end{array}$ \\
\hline $\mathrm{NA}_{4}$ & 312.13 & $\begin{array}{l}7.59-8.59(\mathrm{r}, 4 \mathrm{H} \text {, aromatic ring), } 6.63-7.26 \\
\left(\mathrm{s}, 4 \mathrm{H}, \text { aromatic ring), } 3.04\left(\mathrm{~s}, 2 \mathrm{H}, \mathrm{CH}_{2} \text { in }\right.\right. \\
\text { ring), } 2.70\left(\mathrm{~s}, 6 \mathrm{H}, \mathrm{CH}_{3}\right)\end{array}$ \\
\hline $\mathrm{NA}_{5}$ & 299.10 & $\begin{array}{l}\text { 7.62-8.42 (r, } 4 \mathrm{H} \text {, aromatic ring), } 6.77-7.10 \\
\left(\mathrm{~s}, 4 \mathrm{H}, \text { aromatic ring), } 3.75\left(\mathrm{~s}, 3 \mathrm{H}, \mathrm{CH}_{3}\right),\right. \\
3.24\left(\mathrm{~s}, 2 \mathrm{H}, \mathrm{CH}_{2} \text { in ring) }\right.\end{array}$ \\
\hline $\mathrm{NA}_{6}$ & 283.10 & $\begin{array}{l}\text { 7.62-8.42 (r, } 4 \mathrm{H}, \text { aromatic ring), } 6.99-7.07 \\
(\mathrm{~s}, 4 \mathrm{H}, \text { aromatic ring), } 2.35(\mathrm{~s}, 3 \mathrm{H}, \\
\left.\mathrm{CH}_{3}\right), 3.34\left(\mathrm{~s}, 2 \mathrm{H}, \mathrm{CH}_{2} \text { in ring) }\right.\end{array}$ \\
\hline $\mathrm{NB}_{1}$ & 319.00 & $\begin{array}{l}6.80-7.21(\mathrm{r}, 4 \mathrm{H}, \text { aromatic ring), } 7.09-7.36 \\
(\mathrm{s}, 4 \mathrm{H}, \text { aromatic ring), } 5.67(\mathrm{r}, 1 \mathrm{H}, \mathrm{OH}), \\
3.34\left(\mathrm{~s}, 2 \mathrm{H}, \mathrm{CH}_{2} \text { in ring }\right)\end{array}$ \\
\hline $\mathrm{NB}_{2}$ & 275.05 & $\begin{array}{l}6.76-7.20(\mathrm{r}, 4 \mathrm{H}, \text { aromatic ring }), 7.16-7.22 \\
(\mathrm{~s}, 4 \mathrm{H}, \text { aromatic ring), } 5.66(\mathrm{r}, 1 \mathrm{H}, \mathrm{OH}), \\
3.29\left(\mathrm{~s}, 2 \mathrm{H}, \mathrm{CH}_{2} \text { in ring }\right)\end{array}$ \\
\hline $\mathrm{NB}_{3}$ & 256.09 & $\begin{array}{l}\text { 6.80-7.20 (r, } 4 \mathrm{H} \text {, aromatic ring), } 6.66-7.02 \\
(\mathrm{~s}, 4 \mathrm{H}, \text { aromatic ring), } 5.0(\mathrm{r}, 2 \mathrm{H}, \\
\mathrm{OH}), 3.33\left(\mathrm{~s}, 2 \mathrm{H}, \mathrm{CH}_{2} \text { in ring) }\right.\end{array}$ \\
\hline $\mathrm{NB}_{4}$ & 283.14 & $\begin{array}{l}6.80-7.20(\mathrm{r}, 4 \mathrm{H} \text {, aromatic ring), } 6.52-7.01 \\
(\mathrm{s}, 4 \mathrm{H}, \text { aromatic ring }), 5.0(\mathrm{r}, 1 \mathrm{H}, \mathrm{OH}), 2.85 \\
\left(\mathrm{~s}, 6 \mathrm{H}, \mathrm{CH}_{3}\right), 3.33\left(\mathrm{~s}, 2 \mathrm{H}, \mathrm{CH}_{2} \text { in ring }\right)\end{array}$ \\
\hline $\mathrm{NB}_{5}$ & 270.11 & $\begin{array}{l}6.80-7.20(\mathrm{r}, 4 \mathrm{H}, \text { aromatic ring), } 6.70-7.08 \\
(\mathrm{s}, 4 \mathrm{H}, \text { aromatic ring }), 5.0(\mathrm{r}, 1 \mathrm{H}, \mathrm{OH}), 3.73 \\
\left(\mathrm{~s}, 3 \mathrm{H}, \mathrm{CH}_{3}\right), 3.33\left(\mathrm{~s}, 2 \mathrm{H}, \mathrm{CH}_{2} \text { in ring }\right)\end{array}$ \\
\hline $\mathrm{NB}_{6}$ & 254.11 & $\begin{array}{l}\text { 6.80-7.20 (r, } 4 \mathrm{H} \text {, aromatic ring), 6.99-7.07 } \\
(\mathrm{s}, 4 \mathrm{H}, \text { aromatic ring), } 5.0(\mathrm{r}, 1 \mathrm{H}, \mathrm{OH}), 2.35 \\
\left(\mathrm{s}, 3 \mathrm{H}, \mathrm{CH}_{3}\right), 3.33\left(\mathrm{~s}, 2 \mathrm{H}, \mathrm{CH}_{2} \text { in ring) }\right.\end{array}$ \\
\hline
\end{tabular}

Derivatives $\mathrm{NB}_{2}, \mathrm{NB}_{3}, \mathrm{NB}_{4}$ and $\mathrm{NB}_{6}$ showed greater zone of inhibition than Rifampicin at $10 \mathrm{mg}$ conc. and were the most potent antitubercular derivatives. Derivatives $\mathrm{NA}_{4}$ and $\mathrm{NB}_{1}$ showed greater zone of inhibition than Rifampicin at 10 $\mathrm{mg}$ conc. but showed intermediate anti-tubercular activity at other concentrations. Derivatives $\mathrm{NA}_{1}, \mathrm{NA}_{2}, \mathrm{NA}_{3}, \mathrm{NA}_{5}, \mathrm{NA}_{6}$ and $\mathrm{NB}_{5}$ showed poor antitubercular activity at all concentrations (Table-4).

\begin{tabular}{|c|c|c|c|c|}
\hline \multicolumn{5}{|c|}{$\begin{array}{l}\text { TABLE-4 } \\
\text { TRO ACTIVITY OF SYNTHESIZED } \\
\text { ZOLINE DERIVATIVES }\end{array}$} \\
\hline \multirow[t]{2}{*}{ Compounds } & \multicolumn{4}{|c|}{$\begin{array}{l}\text { Concentration of chemicals (zone of } \\
\text { inhibition in } \mathrm{mm} \text { ) }\end{array}$} \\
\hline & $2.5 \mathrm{mg}$ & $5.0 \mathrm{mg}$ & $7.5 \mathrm{mg}$ & $10.0 \mathrm{mg}$ \\
\hline NA1 & - & 14 & 15 & 17 \\
\hline NA2 & 11 & 14 & 15 & 16 \\
\hline NA3 & - & 12 & 15 & 17 \\
\hline NA4* & 10 & 11 & 14 & 19 \\
\hline NA5 & - & - & 10 & 13 \\
\hline NA6 & - & 11 & 13 & 14 \\
\hline $\mathrm{NB} 1 *$ & 12 & 13 & 15 & 20 \\
\hline $\mathrm{NB} 2 *$ & 18 & 20 & 21 & 22 \\
\hline NB3* & 15 & 17 & 21 & 25 \\
\hline NB4* & 14 & 16 & 25 & 27 \\
\hline NB5 & 12 & 14 & 15 & 17 \\
\hline NB6* & 15 & 17 & 21 & 25 \\
\hline $\begin{array}{l}\text { Standard (Rifampicin) } \\
10 \mathrm{mg}\end{array}$ & \multicolumn{4}{|c|}{18} \\
\hline Control (DMSO) & \multicolumn{4}{|c|}{-} \\
\hline
\end{tabular}

\section{REFERENCES}

1. Rakesh, D.Q. Sun, R.B. Lee, R.P. Tangallapally and R.E. Lee, Eur. J. Med. Chem., 44, 460 (2009).

2. C. Changtam, P. Hongmanee and A. Suksamrarn, Eur. J. Med. Chem, 45, 4446 (2010).

3. M.A. Ali, M. Shaharyar and A.A. Siddiqui, Eur. J. Med. Chem., 42, 268 (2007).

4. P.M. Sivakumar, S.P. Seenivasan, V. Kumar and M. Doble, Bioorg. Med. Chem. Lett., 17, 1695 (2007). 